\title{
STRETCH-INDUCED CONTRACTION OF INTRAFUSAL MUSCLE IN CAT MUSCLE SPINDLE ${ }^{1}$
}

\author{
R. E. POPPELE*,2 AND D. C. QUICK \\ ${ }^{*}$ Laboratory of Neurophysiology and $\ddagger$ Departments of Neurology and Anatomy, University of Minnesota, Minneapolis, Minnesota 55455
}

\begin{abstract}
Measurements of tension, stiffness, and sarcomere length of intrafusal muscle during ramp stretch of isolated muscle spindles have revealed a stretch-induced contraction of the bag 1 fiber. This behavior can account for the very high sensitivity of primary endings to stretch as well as the enhanced sensitivity evoked by dynamic fusimotor stimulation.
\end{abstract}

An intriguing aspect of mammalian muscle spindle behavior is the very high sensitivity of the primary ending compared to that of the secondary ending in the same receptor. There are at least two morphological differences between primary and secondary endings that might account for this difference. The primary ending is formed on the intrafusal bundle in a region called the equator where there are very few myofilaments. The secondary endings are formed in the juxtaequatorial region where there are abundant myofilaments. Thus, the primary nerve terminals lie on muscle that may be more compliant than the muscle under the secondary terminals. With this arrangement, the stretch may induce a greater shearing force between muscle and nerve in the primary area (see Poppele et al., 1979). A second difference is that the intrafusal bundle is composed of three types of muscle (cf., Banks et al., 1977b), and one of these, the bag1 (or "dynamic" bag; Boyd et al., 1975), is innervated by a separate myelinated branch of the primary ending (Banks et al., 1977a). The bag2 (or "static" bag) fiber and the chain fibers are innervated by different myelinated branches of the primary ending and by most of the secondary endings (Boyd, 1962). Since the bag 1 fiber is known to have different mechanical properties from the other two (Boyd, 1976a), the differential sensitivity also might be due in part to mechanical properties that are specific to bag1 fibers.

In this paper, we examine the mechanical properties of the bag1 intrafusal muscle and show that it has a unique reaction to stretching that does, indeed, contribute to the high stretch sensitivity of the primary ending.

\footnotetext{
' This work was supported by Grant BNS 7825165 from the National Science Foundation and Grant NS 10969 from the National Institutes of Health.

${ }^{2}$ To whom correspondence should be addressed at Department of Physiology, University of Minnesota, 435 Delaware Street S. E., Minneapolis, MN 55455.
}

\section{Materials and Methods}

Spindle afferent discharge was recorded from dorsal root filaments of anesthetized cats (pentobarbital sodium, Nembutal, Abbott Laboratories, $35 \mathrm{mg} / \mathrm{kg}$ ). The hindlimb was denervated except for the medial gastrocnemius, and ventral roots L5 through S1 were cut. Activity of single muscle spindle receptors was identified in the usual manner (e.g., Poppele, 1981). Mechanical measurements were made on isolated spindles obtained from tenuissimus muscle taken from anesthetized cats (ketamine hydrochloride, Ketalar, Parke, Davis, $20 \mathrm{mg} /$ $\mathrm{kg}$ ). Details were presented in a previous paper (Poppele et al., 1979).

Isolated spindles. Single spindles were mounted in a small chamber that was perfused continuously with a modified Krebs-Ringer buffer containing amino acids in equilibrium with an atmosphere containing $95 \% \mathrm{O}_{2}$ and $5 \% \mathrm{CO}_{2}$. The spindle was tied at each end to a stiff tungsten wire attached to the shafts of a pair of Pye-Ling model 108 vibrators, each of which was position-servo controlled to provide stretches to the spindle. The chamber was mounted on the stage of a Zeiss photomicroscope equipped with Nomarski interference optics and a 16$\mathrm{mm}$ motion picture camera. Relative stretch by the two pullers could be adjusted to fix the position of any part of the spindle in the microscope field during a stretch. In most cases, the spindle was tied in series with a small wire loop which served as a tension transducer. The opening of the loop was elastic and, therefore, directly proportional to in-series tension.

Experimental protocol. The initial length of the spindle (defined as $L=1.0$ ) was adjusted so that there was just a non-zero tension. The length of the receptor then was measured between the tied ends, and all subsequent stretches were a given fraction of this initial length. Initial lengths were between 3.94 and $4.76 \mathrm{~mm}$. The spindles were stretched to $L=1.05$ times the initial length and a 5\% ramp stretch was applied (i.e., stretch 
from $L=1.05$ to 1.10 ). Ramp duration varied from 2.5 to 4 sec. Intrafusal elements were identified as follows. Chain fibers were identified as those which exhibit kinking for lengths less than $L=1.0$ (Boyd, 1976b). The bag fibers did not show this behavior. Bag1 and bag2 fibers were distinguished from one another by the presence of a "creep" in the bag1 fiber following a ramp stretch (Boyd and Ward, 1969). The "creep" is a slow sliding of intrafusal elements in an otherwise stationary field immediately following the completion of a ramp stretch. Only one of the bag fibers exhibited this behavior and it was taken to be the bagl fiber (Boyd, 1976a; Boyd et al., 1977; Fig. 1).

Recording technique. Data were in the form of motion picture films taken at 14 to 15 frames/sec of different segments of a spindle before, during, and after a ramp stretch. The total field in a single frame was about 175 $\times 250 \mu \mathrm{m}$. These records were projected onto the surface of a two-coordinate digitizer (Graf/Pen Sonic Digitizer, Scientific Accessories Corp.) which provided $x$ and $y$ coordinates to a Heath $\mathrm{H}-11$ laboratory computer. Two basic kinds of measurements were made. Strain, defined as change in length per unit length, was determined by recording the distance between two given points in successive frames. After subtracting the initial distance from each value, they were divided by the initial distance and reported as the percentage of strain. The second type of measurement was a determination of the average sarcomere length in a given muscle fiber. Since the change in observed sarcomere length was less than the resolution of the optical system for measuring any single sarcomere, an average was computed from four consecutive measurements of 20 to 40 sarcomeres. This gave a value whose variability from frame to frame seldom exceeded the range of $\pm 0.025 \mu \mathrm{m}$.

Tension was determined from the in-series wire loop which had a compliance of $5 \mu \mathrm{m} / \mathrm{mg}$. Total stiffness of the receptor, defined as a change in tension per unit length, was determined by superimposing on the ramp stretch a $150-$ msec duration pulse of stretch of magnitude which was one-fourth of the ramp magnitude and measuring the resulting change in tension. If we assume that overall viscosity is affected little by the very slow ramp stretches used, then the viscous component of the tension response to the pulse should be constant and any changes

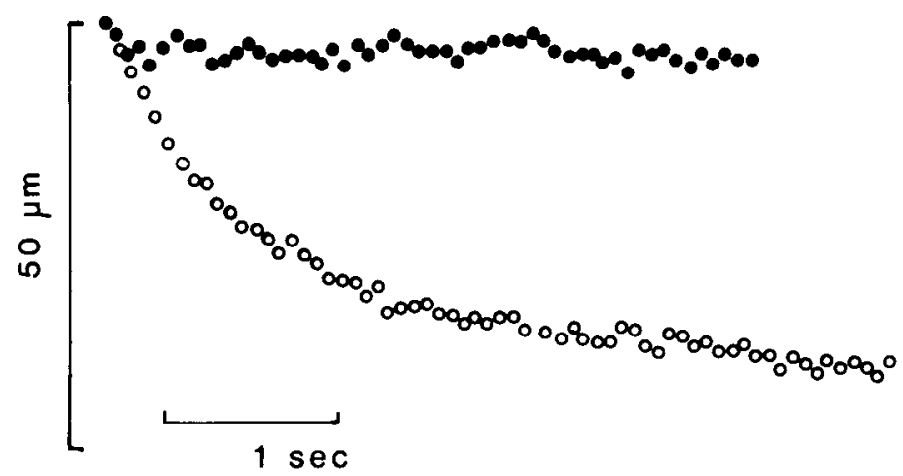

Figure 1. Plot of the movement of a single element in each of the two bag fibers immediately following a ramp stretch. Open circles illustrate the "creep." Solid circles show the behavior of the other bag fiber. Each point represents the measurement for a single frame. observed should be related directly to elastic stiffness. Indeed, stiffness so determined is about the same before and during the first 0.5 to $1 \mathrm{sec}$ of a ramp stretch (see below, Fig. 4).

The analysis was carried out on six isolated spindles. The most complete sets of data from three of those are presented below; partial analysis of the remaining three yielded consistent results.
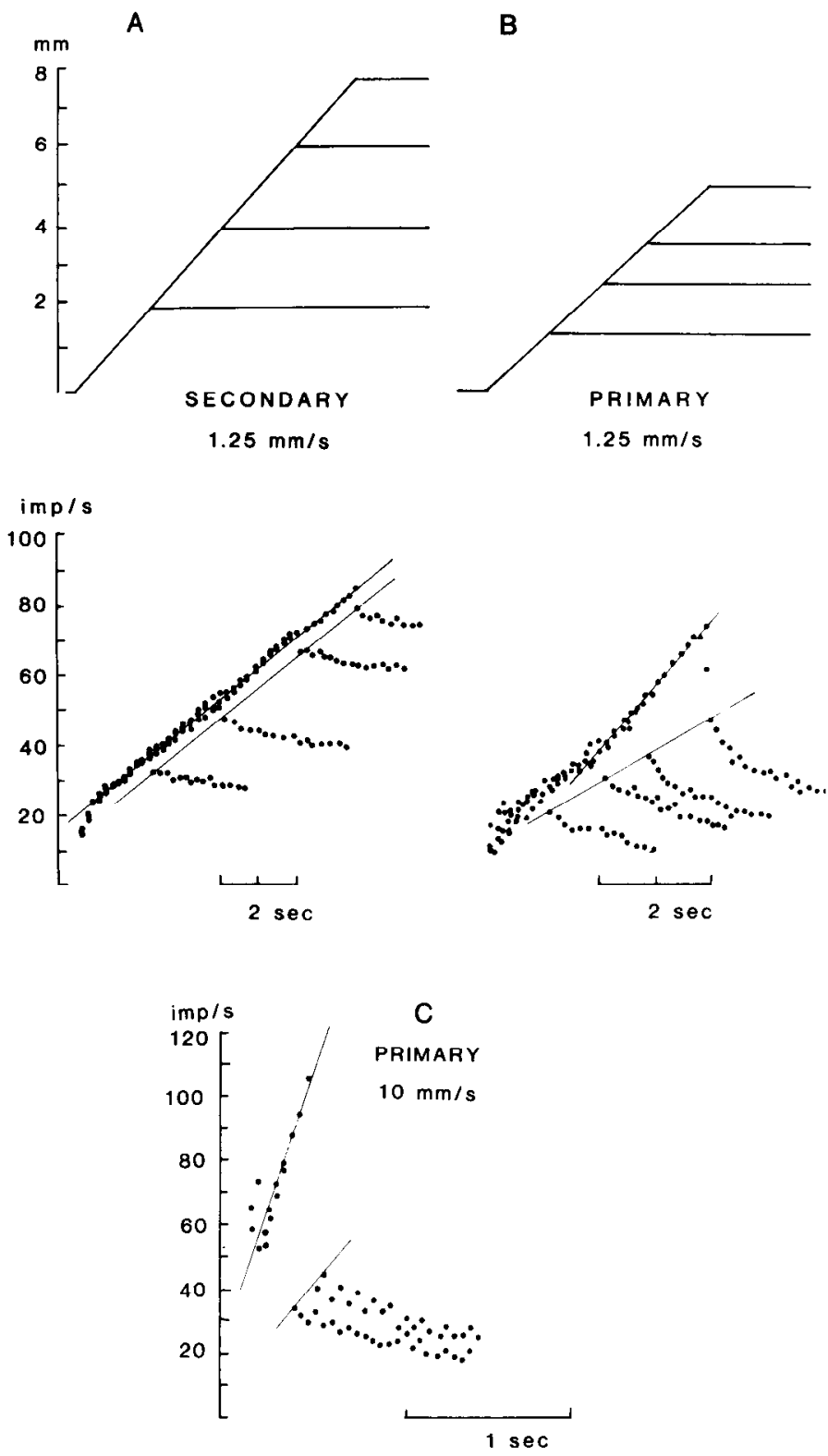

Figure 2. Sensory discharge in a primary and secondary ending in response to ramp stretches. Averaged instantaneous frequency is superimposed for separate stretches of the same velocity but of different magnitudes. Records from medial gastrocnemius spindles are each the average of responses to 6 to 10 stretches. $A$, Secondary ending stretched to four different amplitudes at $1.25 \mathrm{~mm} / \mathrm{sec}$. The slope of discharge rate is 6.4 impulses/sec/mm during the ramp stretch and 6.0 impulses/ $\mathrm{sec} / \mathrm{mm}$ afterward. $B$, Primary ending stretched to four different amplitudes at $1.25 \mathrm{~mm} / \mathrm{sec}$. The slope is 17.5 impulses $/ \mathrm{sec} /$ $\mathrm{mm}$ during the latter part of the stretch and $8.0 \mathrm{impulses} / \mathrm{sec} /$ $\mathrm{mm}$ afterward. $C$, Primary ending stretched to two different amplitudes at $10 \mathrm{~mm} / \mathrm{sec}$. The slope during the stretch is 16.2 impulses $/ \mathrm{sec} / \mathrm{mm}$ and $7.3 \mathrm{impulses} / \mathrm{sec} / \mathrm{mm}$ afterward. 


\section{Results}

The sensitivity of the spindle receptor ending to stretch can be quantitated as the number of impulses per sec evoked per unit of stretch. For a spindle stretched at a constant velocity, the sensitivity during the stretch is given by the slope of the instantaneous frequency (Crowe and Matthews, 1964). By applying a series of stretches at a given velocity but different amplitudes, we also can determine the sensitivity following the stretch by the slope of the incremental activity for each additional amount of stretch in successive records. Application of this technique in Figure $2 \mathrm{~A}$ shows that a secondary ending responds to different amounts of stretch at constant velocity $(1.3 \% / \mathrm{sec})$ with the same sensitivity during and following the stretch; i.e., the two slopes are the same. In contrast, the primary ending responds with a greater sensitivily during a constant velocity stretch than afterward (Fig. $2 B$ ). Furthermore, the change does not appear to depend on the stretch velocity since the sensitivities are about the same for a stretch at 8 times the velocity (Fig. $2 C$ ). With the slower stretches, however, there is a delay in the change of sensitivity occurring during the stretch as evidenced by a change in the slope of the instantaneous frequency part way through the stretch. Therefore, some factor controlling primary, but not secondary, sensitivity during a stretch appears to be altered by the stretch itself.

We have examined the behavior of isolated tenuissimus spindles in order to find a mechanical correlate to this behavior. We applied slow ramp stretches to the isolated receptors and made cinematographic records through Nomarski interference optics. Measurements of strain were made from single frame projections of those records. The data plotted in Figure 3 show strain in the primary sensory area over the bagl fiber and the strain in the adjacent intrafusal muscle. In Figure $3 A$, there was substantial "creep" observed following a stretch. The records in Figure $3 B$ were obtained some time later when the "creep" was no longer evident, a condition indicative of a receptor that is no longer viable (see "Discussion"). In the first records (Fig. $3 A$ ), the strain in the primary sensory area shows the same behavior as the sensory discharge described above, with a shallow initial slope becoming steeper about halfway through the stretch. The muscle fiber outside of the sensory region shows relatively little lengthening except for a yielding just at the end of the stretch. Note also the change of strain distribution between sensory and muscle compartments part way through the stretch. In the latter part of the stretch, proportionally more of the strain is borne by the sensory region. Afterward (in Fig. $3 B$ ), both elements

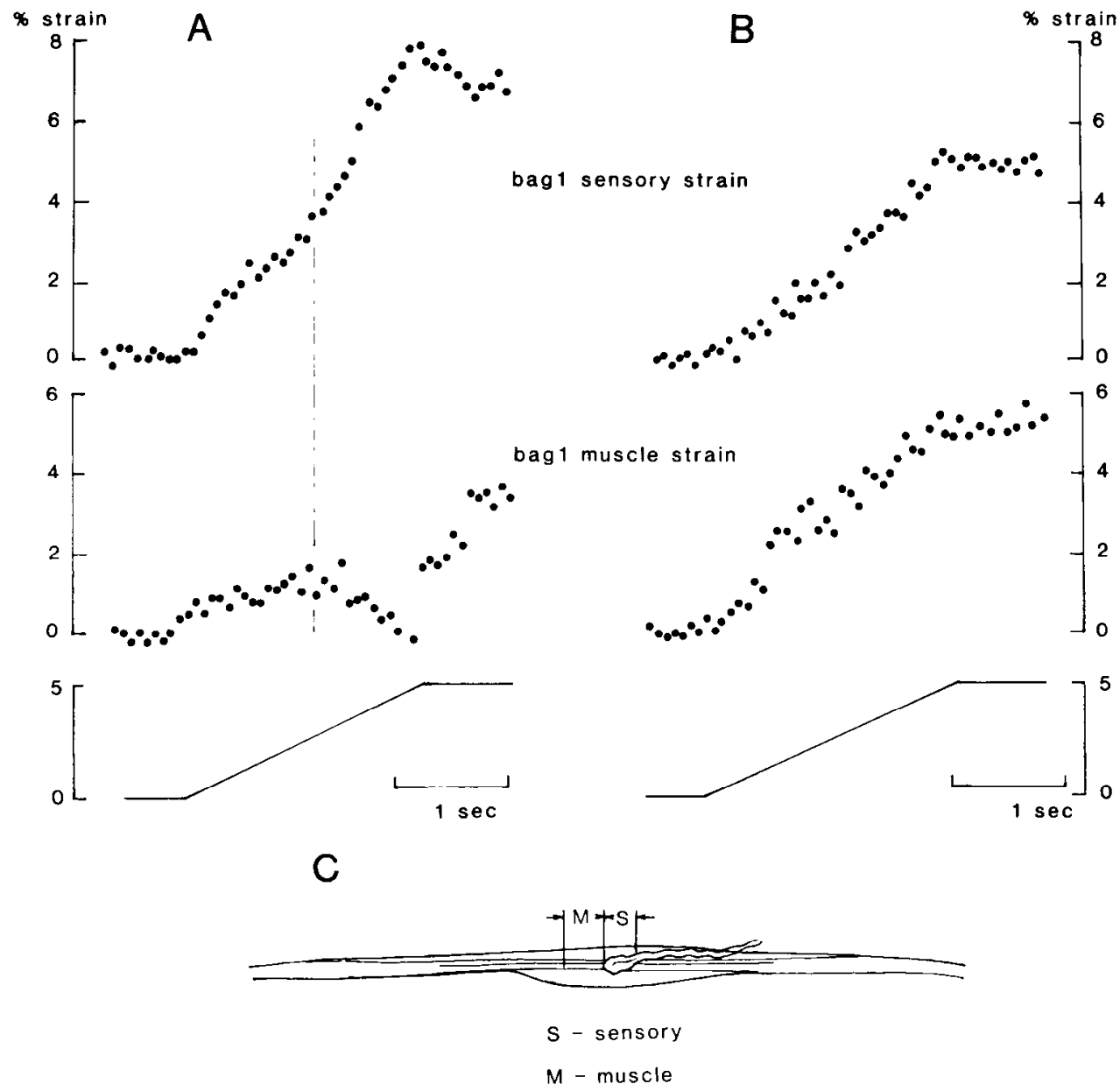

Figure 3. Comparison of sensory and muscle strain in the same spindle before and after the loss of "creep" behavior. $A$, Response while "creep" was evident; dashed line indicates the onset of change in the distribution of strain between sensory and muscle areas. $B$, Response when "creep" was no longer evident. $C$, Tracing of the spindle and primary ending to show where the measurements were made. 
were stretched about equally with no obvious change in slope or distribution.

We may conclude from these observations that some process associated with the healthy spindle changes the stiffness of some part of the receptor in the course of the stretch. We cannot determine, though, whether the sensory area becomes more compliant or whether the intrafusal muscle becomes more stiff. The data from another spindle presented in Figure 4 show, however, that the change is associated with an increase in stiffness. Total tension in the spindle shows an increase in the slope, and total stiffness, as determined by tension response to pulses of stretch, increases in the latter half of the stretch.
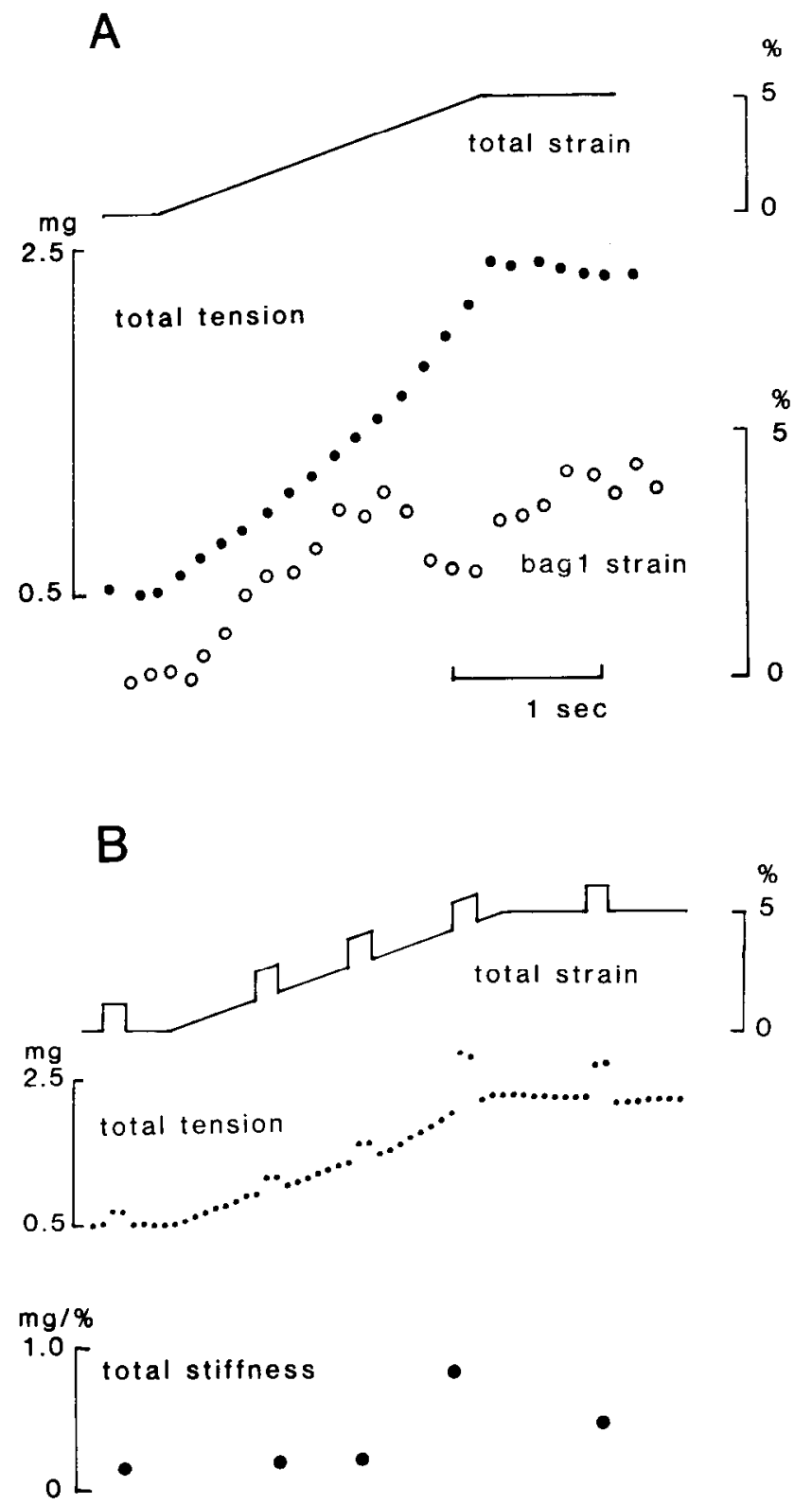

Figure 4. Comparison of total spindle tension, bag1 strain, and total spindle stiffness. $A$, Tension and bag1 strain response to a $5 \%$ ramp stretch. $B$, Tension response to the same ramp with superimposed stretch pulses. Spindle stiffness is reported as milligrams of tension per $1 \%$ stretch and was calculated from the tension response to the applied pulses as explained in the text. 'The time calibration bar applies to $A$ and $B$.
At the same time, the bag 1 intrafusal strain in the juxtaequatorial region is seen to decrease.

In Figure 5, we have plotted the average sarcomere length in the bag1 and the bag2 intrafusal muscle from a third spindle to show that the decreased strain noted above is due to a shortening of sarcomeres in the bag 1 fiber. Note that the bag2 fiber does not exhibit this behavior and that its sarcomeres appear to be stretched passively by the stimulus.

\section{Discussion}

We conclude from the data presented above that there is a stretch-activated contraction of the bag1 intrafusal muscle. The key observation supporting this conclusion is the increased slope of spindle tension that is coincident with the decreased slope of bagl muscle strain. An increasing slope of tension with a constant rate of stretch is a clear indication of increasing stiffness. Although spindle stiffness is expected to increase due to the static length-tension properties of the intrafusal muscle, stiffness is nearly constant for lengths less than 1.10 times the rest length (see Poppele et al., 1979). Furthermore, Figure 4 shows that stiffness is much greater in the latter half of the ramp than following it, even though spindle length is greater when the stretch is completed. The observed shortening of bag 1 sarcomeres with an increasing tension (Fig. 5) confirms the contraction in this fiber.

The "creep" that is observed following a stretch is
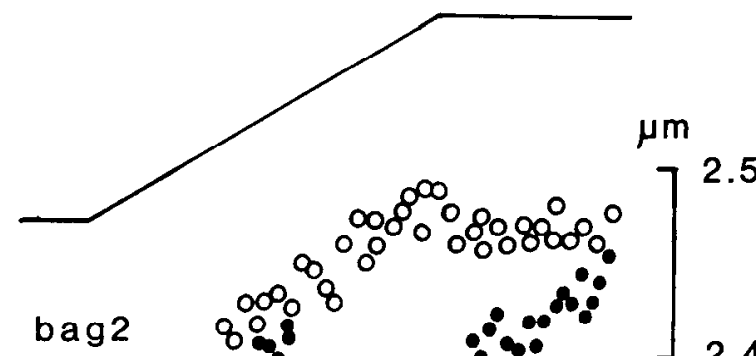

2.4

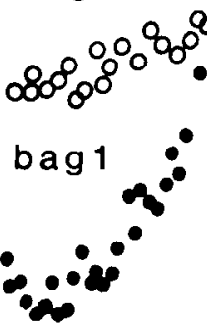

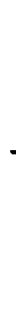

$\mathrm{mg}$
0.6

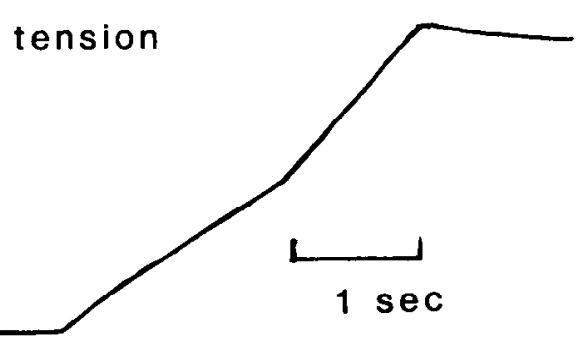

Figure 5. Sarcomere lengths in bag fibers during a ramp stretch. Open circles are from bag2 and solid circles are from bag1 measurements. Total spindle strain (top trace) and total spindle tension (bottom trace) are plotted for reference. 
associated with a lengthening of bag1 sarcomeres and, therefore, is a slow relaxation of the stretch-induced contraction. Thus, it is consistent that no contraction is observed when there is no longer a "creep" (Fig. $3 B$ ). Furthermore, in unmodified Krebs-Ringer buffer, "crecp" behavior is rarely seen and intrafusal contractions cannot be evoked consistently by fusimotor stimulation (see Boyd and Ward, 1975; Poppele et al., 1979). The decay of sensory discharge in the primary ending following a stretch has the same time course as the "creep" (compare Figs. 1 and $2 B$ ). We have observed that this sensory adaptation following a stretch can be cut short by a brief pulse of stretch (Fig. 6A). The same behavior is observed in the tension record (Fig. $6 B$ ), thus suggesting that bonds that are formed by the stretch activation may be broken by a sudden mechanical perturbation. We note that the effect in the tension record is much smaller than the effect observed in the sensory discharge. This is undoubtedly due to the fact that the total tension is a weighted sum of the tensions in each of the individual muscle fibers as well as the capsule, not only that of bag1, whereas the receptor may be responding primarily to forces set up by the bagl.

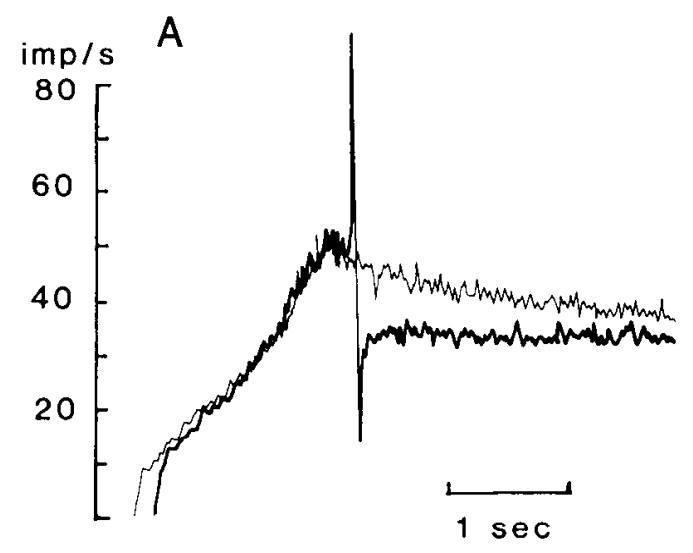

B

\%

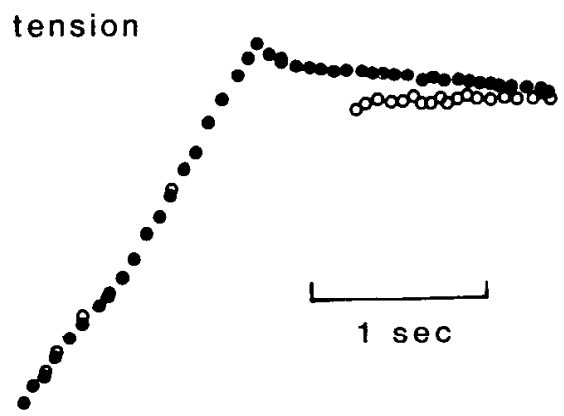

Figure 6. Effect of a pulse of stretch applied just after a ramp stretch. $A$, Sensory discharge with and without the pulse for successive trials. $\mathrm{imp} / \mathrm{s}$, impulses per sec. $B$, Tension recurd from another spindle using same protocol. Open circles are from tension measurements when the pulse was applied and solid circles are from measurements when it was not.
It was noted by Crowe and Matthews (1964) that the slope of the response to a ramp stretch is increased by dynamic axon stimulation but not by static axon stimulation. Such stimulation has been observed to produce very small contractions (a 3\% local shortening for stimulation frequencies of $110 / \mathrm{sec}$ ) in the polar regions of bag1 fibers but no contraction in the juxtaequatorial region (Bessou and Pagès, 1975). Because the direct effect of such stimulation seems so small, its dramatic effect on the sensitivity of the primary ending has been a mystery. It may be that the effect of dynamic fusimotor stimulation on this fiber is to potentiate the stretchactivated contraction. In fact, single shock stimulation of dynamic efferent fibers evokes a sensory response in the primary ending only if it is applied during a stretch (Laporte et al., 1981). Therefore, the dynamic $\gamma$ activation of spindles may be explained as an enhanced increase of sensitivity to dynamic stretching. This leads to a greater dynamic index (i.e., the difference in firing rate at the peak of a stretch and that recorded $0.5 \mathrm{sec}$ later) because there would be a greater relaxation from an enhanced stretch-activated contraction.

The absence of such behavior in the other intrafusal muscle fibers is evidenced by the absence of "creep" and the absence of the shortening of sarcomeres during stretch. Furthermore, activation of these fibers by static axon stimulation does not appreciably change the slope of primary ending responses to stretch.

The term "stretch activation" used here to describe the reaction of bag 1 intrafusal fibers to an imposed stretch is taken from the literature on insect fibrillar muscle (cf., Pringle, 1978). In the flight muscles of certain beetles, stretch-activated contractions cause shortening which, in turn, stretches the antagonist muscle, causing it to contract. A cyclic flight motion then is produced by the alternate contraction of these two muscles (Pringle, 1949). Vertebrate skeletal muscle (e.g., the psoas muscle of the rabbit; Rüegg et al., 1970) and cardiac muscle (Steiger, 1971) show the same phenomenon, but the stretch activation in these cases is subtle and seems to have only a negligible effect on muscle length. In the case of intrafusal muscle, the shortening by stretch activation extends the sensory endings and thus adds to the applied stretch. In addition, the increased stiffness of in-series

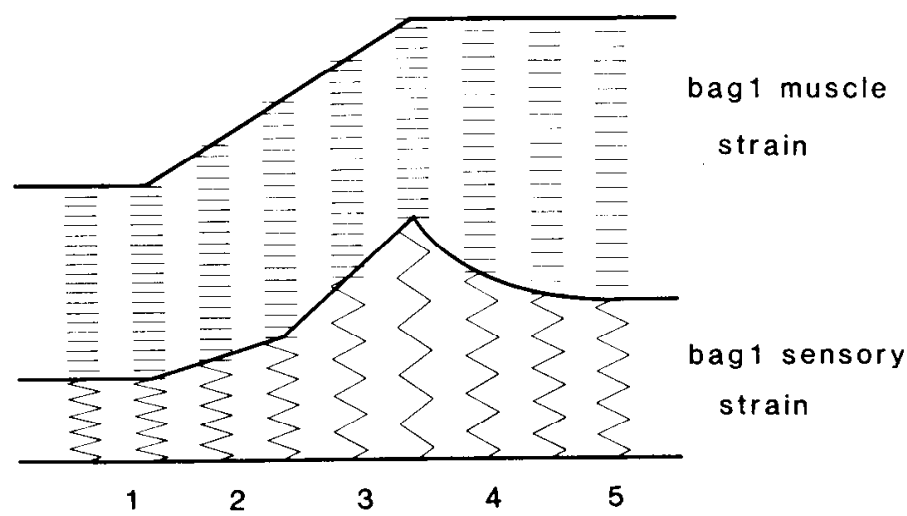

Figure 7. Diagram of the partitioning of strain between bag1 intrafusal muscle and primary sensory endings during a slow ramp stretch. See the text for details. 
muscle leads to a greater stretching of the primary sensory area and, therefore, a greater sensitivity. This is illustrated diagrammatically in Figure 7. Initially, in seg. ment 1 , there is a partitioning of strain between bag 1 intrafusal muscle and the primary area according to the relative stiffness of these two elements (see Fig. 8 of Poppele et al., 1979). As a slow stretch is applied (segment 2 of Fig. 7), there is no initial change in stiffness and the relative partitioning of strain remains the same. Part way through the stretch (segment 3), the bag1 muscle stiffness increases and there is a slight shortening of the bag 1 fiber. This leads to a relatively greater strain in the sensory area. Relaxation of the bag1 fiber following the stretch is accompanied by a decreasing stiffness and a lengthening of that element (segment 4), leading to a shortening (adaptation) in the sensory area. This continues for several seconds until a new steady state partitioning is established in segment 5 .

The delayed onset of stretch activation reported here is probably due to the very slow stretches used (1.25 to $2.0 \% / \mathrm{sec}$ ). However, as seen in Figure $2 C$, there is an immediate increase of primary ending sensitivity lasting the entire duration of a stretch if the stretch is applied more rapidly. Also, in a previous paper describing the behavior of spindles to randomly applied stretches of various velocities and durations, we noted that "a 'dynamic' nonlinearity affecting only primary endings ...depends on the duration of an applied stretch" (Poppele, 1981). It now seems that the nonlinearity is a stretch-activated contraction of the bag1 intrafusal muscle.

\section{References}

Banks, R. W., D. Barker, and M. J. Stacey (1977a) Intrafusal branching and distribution of primary and secondary afferents. J. Physiol. (Lond.) 272: 66P-67P.

Banks, R. W., D. W. Harker, and M. J. Stacey (1977b) A study of mammalian intrafusal muscle fibers using a combined histochemical and ultrastructural technique. J. Anat. 123: 783-796.

Bessou, P., and B. Pagés (1975) Cinematographic analysis of contractile events produced in intrafusal muscle fibers by stimulation of static and dynamic fusimotor axons. J. Physiol. (Lond.) 252: 397-427.
Boyd, I. A. (1962) The structure and innervation of the nuclear bag muscle fibres system and the nuclear chain muscle fibre system in mammalian muscle spindle. Philos. Trans. R. Soc. Lond. (Biol.) 245: 81-136.

Boyd, I. A. (1976a) The mechanical properties of dynamic nuclear bag fibres, static nuclear bag fibres and nuclear chain fibres in isolated cat muscle spindles. Prog. Brain Res. 44: $33-50$.

Boyd, I. A. (1976b) The response of fast and slow nuclear bag fibres and nuclear chain fibres in isolated cat muscle spindles to fusimotor stimulation, and the effect of intrafusal contraction on the sensory endings. Q. J. Exp. Physiol. 61: 203-252.

Boyd, I. A., and J. Ward (1969) The response of isolated cat muscle spindles to passive stretch. J. Physiol. (Lond.) 200: 104-105.

Boyd, I. A., and J. Ward (1975) Motor control of nuclear bag and nuclear chain fibres in isolated living muscle spindles from the cat. J. Physiol. (Lond.) 244: 83-112.

Boyd, I. A., M. H. Gladden, P. N. McWilliam, and J. Ward (1975) 'Static' and 'dynamic' nuclear bag fibres in isolated cat muscle spindles. J. Physiol. (Lond.) 250: 11P-12P.

Boyd, I. A., M. H. Gladden, P. N. McWilliam, and J. Ward (1977) Control of dynamic and static nuclear bag fibres and nuclear chain fibres by gamma and beta axons in isolated cat muscle spindles. J. Physiol. (Lond.) 265: 133-162.

Crowe, A., and P. B. C. Matthews (1964) Further studies of static and dynamic fusimotor fibers. J. Physiol. (Lond.) 174. $132-151$

Laporte, Y., F. Emonet-Dénand, and F. Jami (1981) The skeletomotor or $\beta$-innervation of muscle spindles. Trends Neurosci. 4: 97-99.

Poppele, R. E. (1981) An analysis of muscle spindle behavior using randomly applied stretches. Neuroscience $6: 1157-1165$.

Poppele, R. E., W. R. Kennedy, and D. C. Quick (1979) A determination of static mechanical properties of intrafusal muscle in isolated cat muscle spindles. Neuroscience 4: 401411.

Pringle, J. W. S. (1949) The excitation and contraction of the flight muscles of insects. J. Physiol. (Lond.) 108: 226-232.

Pringle, J. W. S. (1978) Stretch activation of muscle: Function and mechanism. Proc. R. Soc. Lond. (Biol.) 201: 107-130.

Rüegg, J. C., G. J. Steiger, and M. Schädler (1970) Mechanical activation of the contractile system in skeletal muscle. Pfluegers Arch. 319: 139-145.

Steiger, G. J. (1971) Stretch activation and myogenic oscillation of isolated contractile structures of heart muscle. Pfluegers Arch. 330: 347-361. 